\title{
Precision Environmental Radiation Monitoring System
}

\author{
Vladimir Popov, Pavel Degtiarenko \\ Thomas Jefferson National Accelerator Facility, Newport News, Virginia, USA 23606
}

A new precision low-level environmental radiation monitoring system is being developed and tested
at Jefferson Lab, capable of long-term environmental radiation measurements with accuracy and
stability of the order of $1 \mathrm{nSv} / \mathrm{h}$ in an hour, roughly corresponding to approximately $1 \%$ of the natural
cosmic background at sea level. Advanced highly sensitive electronic front-end has been developed
for use with the industry-standard Reuter-Stokes RS-1013 and some older models High Pressure
lonization Chamber detector hardware. Ionization chambers (IC) are widely used for accurate ionization radiation monitoring because of outstanding long term stability, but their major drawback is a relatively low output signal. The IC consists of a $25 \mathrm{~cm}$ diameter stainless steel vessel filled high purity argon gas at $25 \mathrm{~atm}$. At normal conditions it produces 200-500 fA output current from natural background radiation in the US costal region. Measuring of hundreds $f A$ current with accuracy and stability in the range of few $f A$ in the conditions when the device has to operate continuously outside in the field in the range of environmental temperatures $\left(-10\right.$ to $\left.+40^{\circ} \mathrm{C}\right)$ is still difficult. Many of known solutions based on different DC readout interfaces are subject to errors and instability due to parasitic DC current leakages. Problems with DC offsets and errors may be solved using chopping amplifiers that convert $D C$ to $A C$ and due to this conversion most of DC leakages and offsets are eliminated. Several such devices tested in practice and used in the industry, like Cary Instruments "Vibrating Reed Electrometer", work very well for measurements of sub-femtoampere currents, but they cannot be used with the Reuter-Stokes ionization chambers because of the relatively high $100 \mathrm{pF}$ output capacitance of the chamber. The solution for stable and reliable low current monitoring using precision low leakage reed relay was invented and tested at the Jefferson Lab's Radiation Control Group. Our electronic circuit is designed to collect charge from the virtually suspended ionization chamber ion collecting electrode (cathode). The cathode is disconnected from the charge sensing amplifier most of the time (over $99.9 \%$ duty factor) using a low leakage and low capacitance reed relay. The ionization charge is accumulated at the cathode, charging the chamber's output capacitance. The low leakage precision reed relay is powered by the voltage source that generates low frequency ramp-shaped balanced pulses that reduce to minimum the crosstalk noise from the relay coil operating current. At the moment the relay contacts close at the top of the ramp, the accumulated charge is transmitted from the IC cathode to the amplifier circuit during a $0.25 \mathrm{~ms}$ time interval, then the relay control voltage is reset, and the relay opens again to start the new charge collecting cycle. AC coupled charge sensitive amplifier with subsequent dynamic pulse amplitude capture circuit allowed to reduce to minimum all possible constant pedestal and bias effects in the readout circuit. A normal integration time period is about one second to preserve a reasonably fast reaction to ionization changes. Correspondingly, the input circuit's dead time is only $0.025 \%$. Each cycle, the output buffer amplifier generates a pulse of a standard shape and width of the order of $0.5 \mathrm{~ms}$, with the pulse height proportional to the charge collected during the cycle. Such pulses can be transmitted over long distances through regular telephone cable or RF cable lines. New method of data acquisition has been developed and tested together with the new readout. It allowed accurate and stable continuous data collection in a remote Linux-operated computer workstation, connected to the detectors using standard telephone cable line and off-the-shelf analog to digital signal processing hardware. The data acquisition system algorithm is built around the continuously running 24 bit resolution $192 \mathrm{kHz}$ data sampling analog to digital convertor. The data flow is analyzed online in real time by the signal recognition, analysis, and accumulation software based on the open-source Physics Analysis Workstation (PAW) software package from CERN. The analysis routine decodes pulse amplitudes and determines time intervals between pulses. Same program provides a parallel extraction and record of temperature information from a temperature sensor installed inside the detector, the information being encoded in the same data flow. All pulse height, time interval, and temperature data are accumulated continuously and stored in the workstation for subsequent analysis and history presentation. Charge accumulated over one period characterizes average current measured. The major features of the design include: extremely low leakage current in the input circuit limited only by connector insulation where resistance is greater than $10^{12}$ ohms, true charge integrating mode operation, relatively fast response to the intermediate radiation change, and high degree of thermal and long-term stability. These features allow using the device as an environmental radiation monitor at the perimeters of the radiation-generating installations in densely populated areas, and in other monitoring and security applications requiring high precision and long-term stability. Initial system evaluation results are presented, illustrating radiation background measurements at the Jefferson Lab CEBAF accelerator 
site during and between accelerator operations. Future full system implementation as well as possible general applications of the new front-end electronics design for precision ultra-low current measurement and monitoring systems will be discussed.

Notice: Authored by Jefferson Science Associates, LLC under U.S. DOE Contract No. DE-AC0506OR23177. The U.S. Government retains a non-exclusive, paid-up, irrevocable, world-wide license to publish or reproduce this manuscript for U.S. Government purposes.

\section{* Abstract Submittal Form *}

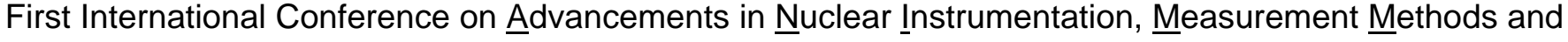
their Applications

- ANIMMA -

Session Topics: Fundamental Physics

Prefer: [Choose one from these 3 options]

冈 Oral Session $\square$ Poster Session $\quad \square$ No Preference

Family name: Popov First name: Vladimir

Affiliation/Institution/Company: Thomas Jefferson National Accelerator Facility

Mailing Address: 12050 Jefferson Ave, Suite 602

City: Newport News

State/Province: Virginia

Zip Code: 23606

Country: USA

Phone: 7572696360

Fax:

E-mail: popov@jlab.org

Abstract must be received no later than Sunday, January 18, 2009.

Abstract must be in MS-Word and PDF only and must not exceed 1000 words.

Please e-mail abstract to: animma@cea.fr 